\title{
Coherent vortices in high resolution direct numerical simulation of homogeneous isotropic turbulence: A wavelet viewpoint
}

\author{
Naoya Okamoto ${ }^{\text {a) }}$ and Katsunori Yoshimatsu ${ }^{\text {b) }}$ \\ Department of Computational Science and Engineering, Nagoya University, Nagoya 464-8603, Japan \\ Kai Schneider ${ }^{\mathrm{c})}$ \\ MSNM-CNRS and CMI, Université de Provence, 39 rue Frédéric Joliot-Curie, \\ 13453 Marseille Cedex 13, France \\ Marie Farge ${ }^{\mathrm{d})}$ \\ LMD-IPSL-CNRS, Ecole Normale Supérieure, 24 rue Lhomond, 75231 Paris Cedex 05, France \\ Yukio Kaneda ${ }^{\mathrm{e}}$ \\ Department of Computational Science and Engineering, Nagoya University, Nagoya 464-8603, Japan
}

(Received 24 January 2007; accepted 11 July 2007; published online 19 November 2007)

\begin{abstract}
Coherent vortices are extracted from data obtained by direct numerical simulation (DNS) of three-dimensional homogeneous isotropic turbulence performed for different Taylor microscale Reynolds numbers, ranging from $\operatorname{Re}_{\lambda}=167$ to 732 , in order to study their role with respect to the flow intermittency. The wavelet-based extraction method assumes that coherent vortices are what remains after denoising, without requiring any template of their shape. Hypotheses are only made on the noise that, as the simplest guess, is considered to be additive, Gaussian, and white. The vorticity vector field is projected onto an orthogonal wavelet basis, and the coefficients whose moduli are larger than a given threshold are reconstructed in physical space, the threshold value depending on the enstrophy and the resolution of the field, which are both known a priori. The DNS dataset, computed with a dealiased pseudospectral method at resolutions $N=256^{3}, 512^{3}, 1024^{3}$, and $2048^{3}$, is analyzed. It shows that, as the Reynolds number increases, the percentage of wavelet coefficients representing the coherent vortices decreases; i.e., flow intermittency increases. Although the number of degrees of freedom necessary to track the coherent vortices remains small (e.g., $2.6 \%$ of $N$ $=2048^{3}$ for $\operatorname{Re}_{\lambda}=732$ ), it preserves the nonlinear dynamics of the flow. It is thus conjectured that using the wavelet representation the number of degrees of freedom to compute fully developed turbulent flows could be reduced in comparison to the standard estimation based on Kolmogorov's theory. (C) 2007 American Institute of Physics. [DOI: 10.1063/1.2771661]
\end{abstract}

\section{INTRODUCTION}

Direct numerical simulation (DNS) of homogeneous isotropic turbulence using Fourier spectral methods has a long tradition. Starting in the late 1960 s with $32^{3}$ simulations, ${ }^{1,2}$ the increasing CPU speed and memory of supercomputers meanwhile allow simulations up to $4096^{3}$ grid points and $\operatorname{Re}_{\lambda}=1201$ performed on the Earth Simulator (ES). ${ }^{3,4}$ The series of high resolution DNS datasets obtained on the ES suggests that the normalized mean energy dissipation per unit mass tends to a constant and hence becomes independent of the kinematic viscosity as the viscosity tends to zero. Furthermore, it has been shown that the scaling of the energy spectrum is about 0.1 steeper than the Kolmogorov scaling of $-5 / 3$ and presents a bottleneck at high wavenumbers. ${ }^{4}$ The number of dynamically active (spatial and temporal) scales involved in the simulations increases strongly with the

\footnotetext{
${ }^{a)}$ Electronic mail: okamoto@ fluid.cse.nagoya-u.ac.jp

${ }^{\text {b) }}$ Author to whom correspondence should be addressed. Electronic mail: yosimatu@fluid.cse.nagoya-u.ac.jp

c)Electronic mail: kschneid@cmi.univ-mrs.fr

${ }^{\mathrm{d})}$ Electronic mail: farge@ @lmd.ens.fr

${ }^{\text {e) }}$ Electronic mail: kaneda@cse.nagoya-u.ac.jp
}

Reynolds number. A dimensional analysis based on the Kolmogorov scaling predicts $N \propto \operatorname{Re}_{\lambda}^{9 / 2}$, where $N$ denotes the total number of spatial grid points. Since the frontiers of DNS are limited by the available computational resources, the computation of high Reynolds number flows, as needed in most computational fluid dynamics applications, still requires ad hoc turbulence models.

Starting from random initial conditions, a typical feature of all these flows is their self-organization and the formation of regions of intense vorticity, as predicted by Taylor ${ }^{5}$ and observed in both numerical ${ }^{6-9}$ and laboratory ${ }^{10}$ experiments. For a given flow realization, these coherent structures are not homogeneously distributed, neither in space nor in time. This suggests the possibility of flow intermittency, defined as the lacunarity of the fine scale activity, which means that the spatial support of the active regions is decreasing with scale. To be able to benefit from this property, a suitable representation of the flow should take into account this lacunarity, in both space and time.

Multiscale decompositions, computed efficiently by fast wavelet transforms, allow a sparse representation of intermittent data. The wavelet transform decomposes a given flow field into scale-space contributions from which it is recon- 
structed. If the flow is intermittent, the small scale contributions have significant values only in active regions and are nonsignificant in weak regions. Hence, the nonsignificant contributions can be neglected and the amount of wavelet coefficients can be significantly reduced before reconstructing the flow field in physical space.

Wavelet techniques to analyze turbulent flows were pioneered at the end of the 1980s. ${ }^{11}$ Since then these techniques have been further developed and exploited for analyzing experimental and numerical data, to model turbulent flows and also for solving the Navier-Stokes equations directly in wavelet space. A review is beyond the scope of the paper (see further details in Refs. 12-15).

We have introduced a wavelet-based coherent vortex extraction technique for two-dimensional flows using scalarvalued orthogonal wavelet decompositions. ${ }^{16}$ The algorithm is based on an orthogonal wavelet decomposition of the vorticity. A threshold motivated from denoising theory ${ }^{17}$ and without any adjustable parameters allows to split the wavelet coefficients into two sets. A subsequent reconstruction in physical space from both coefficient sets yields a separation of the vorticity field into two orthogonal parts. The coherent vorticity reconstructed from few wavelet coefficients, whose moduli are above the threshold, contains the coherent vortices, most of the energy, and exhibits statistics similar to the total vorticity. The incoherent vorticity reconstructed from most of the wavelet coefficients, whose moduli are below the threshold, corresponds to an almost uncorrelated random background flow with quasi-Gaussian one-point statistics. This technique has been extended to three-dimensional (3D) flows using a vector-valued orthogonal wavelet decomposition. ${ }^{18}$ We applied it to DNS data computed at resolution $256^{3}$ corresponding to a Taylor microscale Reynolds number $\operatorname{Re}_{\lambda}=150$. We showed that only $3 \%$ of the wavelet coefficients, the strongest ones, represent the coherent vortex tubes which retain $99 \%$ of the energy and $75 \%$ of the enstrophy of the flow. Coherent vortex extractions using biorthogonal wavelet decompositions have been presented in Refs. 19 and 20. In Ref. 21, we proposed a new turbulence model, called coherent vortex simulation (CVS), which is based on deterministic computation of the coherent flow evolution, and modeling of the influence of the incoherent background flow. Applications of CVS to two-dimensional flows and to three-dimensional turbulent mixing layers can be found in Refs. 22 and 23, respectively.

The aim of the present paper is to apply the coherent vortex extraction algorithm to higher resolution DNS data of homogeneous isotropic turbulence (up to $\mathrm{Re}_{\lambda}=732$ ) in order to study the influence of the Reynolds number. The key question for the feasibility of the CVS approach is to estimate how the number of retained coefficients $N_{c}$, representing the coherent flow, depends on the Reynolds number. As mentioned above, the statistical theory of turbulence suggests a scaling in $N \propto \operatorname{Re}_{\lambda}^{\zeta}$ with $\zeta=9 / 2$ for DNS, while in a recent paper $^{24}$ an even less optimistic exponent of $\zeta=6$ has been recommended to guarantee well resolved DNS. If our analyses show a slower increase of $N_{c}$ with $\operatorname{Re}_{\lambda}$ than the increase of $N$ with $\operatorname{Re}_{\lambda}$, i.e., $N \propto \operatorname{Re}_{\lambda}^{9 / 2}$, CVS may become more inter- esting for computing high Reynolds number flows than standard methods.

The organization of the present paper is as follows. After a short review of wavelet-based analysis tools and coherent vortex extraction in Sec. II, we present the parameters of the DNS data and show some flow visualizations in Sec. III. In Sec. IV we apply the coherent vortex extraction to a flow for $\operatorname{Re}_{\lambda}=732$. We analyze the total, coherent, and incoherent flows in physical space and investigate their statistical behavior. We also study the energy transfers and fluxes in spectral space. In Sec. V the dependence of the percentage of wavelet coefficients representing the coherent vorticity (compression rate) on the Reynolds number is shown and we estimate how the number of coefficients representing the coherent part of the flow depends on the Reynolds number. Finally, conclusions are drawn in Sec. VI and some perspectives for the CVS based on the orthogonal wavelet decomposition are presented. Appendix A discusses technical questions on the influence of the divergence of the vector-valued wavelet basis. In Appendix B, the influence of the number of the iterations in the coherent vortex extraction method is examined.

\section{WAVELET ANALYSIS AND COHERENT VORTEX EXTRACTION}

For definitions and details on the orthogonal wavelet transform, its extension to higher dimensions, we refer the reader to, e.g., Refs. 12 and 25. In this section, we fix the notation for the orthogonal wavelet decomposition of a three-dimensional vector-valued field. We define moments of the wavelet coefficients that allow scale-dependent statistics and summarize the main ideas of the coherent vortex extraction method.

\section{A. Vector-valued orthogonal wavelet decomposition}

We consider a vector field $\boldsymbol{v} \in L^{2}\left(\mathbf{R}^{3}\right)$ sampled on $N$ $=2^{3 J}$ equidistant grid points, $J$ being the number of octaves in each space direction. The wavelet transform unfolds $\boldsymbol{v}$ into scale, positions, and directions using a function $\psi$, called the "mother wavelet." A wavelet is well-localized in space $\boldsymbol{x}$ $\in \mathbf{R}^{3}$ (i.e., it exhibits a fast decay for $|\boldsymbol{x}|$ tending to infinity), is oscillating (i.e., $\psi$ has at least a vanishing mean value, or better, the first $m$ moments of $\psi$ vanish), and is smooth (i.e., its Fourier transform $\mathcal{F}[\psi](\boldsymbol{k})$ exhibits fast decay for wavenumbers $|\boldsymbol{k}|$ tending to infinity). The mother wavelet then generates a family of wavelets $\psi_{\lambda}(\boldsymbol{x})$, by dilation, translation, and rotation, which yields an orthogonal basis of $L^{2}\left(\mathbf{R}^{3}\right)$.

The vector field $\boldsymbol{v}$ can be decomposed into an orthogonal wavelet series

$$
\boldsymbol{v}(\boldsymbol{x})=\sum_{\lambda \in \Lambda} \widetilde{\boldsymbol{v}}_{\lambda} \psi_{\lambda}(\boldsymbol{x})
$$

where the multi-index $\lambda=\left(j, i_{x}, i_{y}, i_{z}, \mu\right)$ denotes the scale $j$, the position $\boldsymbol{i}=\left(i_{x}, i_{y}, i_{z}\right)$, and the seven directions $\mu$ $=1, \ldots, 7$ of the wavelets. The index set $\Lambda$ is 


$$
\begin{aligned}
\Lambda= & \left\{\lambda=\left(j, i_{n}, \mu\right), j=0, \ldots, J-1, i_{n}=0, \ldots, 2^{j}-1,\right. \\
& n=1,2,3, \text { and } \mu=1, \ldots, 7\} .
\end{aligned}
$$

The index set $\Lambda$ corresponds to the octree representation of the orthogonal wavelet coefficients (which is the generalization to $3 \mathrm{D}$ of the dyadic tree in one dimension). Consequently, there are more coefficients when scale decreases (i.e., $j$ increases). Due to orthogonality, the wavelet coefficients are given by $\widetilde{\boldsymbol{v}}_{\lambda}=\left\langle\boldsymbol{v}, \psi_{\lambda}\right\rangle$, where $\langle\cdot, \cdot\rangle$ denotes the $L^{2}$ inner product, defined by $\langle f, g\rangle=\int_{\mathbf{R}^{3}} f(\boldsymbol{x}) g(\boldsymbol{x}) d \boldsymbol{x}$. The coefficients measure the fluctuations of $\boldsymbol{v}$ around scale $2^{-j}$ and around position $i / 2^{j}$ in one of the directions. The $N$ wavelet coefficients $\widetilde{\boldsymbol{v}}_{\lambda}$ are efficiently computed from the $N$ grid point values of $\boldsymbol{v}$ using the fast wavelet transform, which has linear complexity. ${ }^{25}$

\section{B. Scale-dependent moments}

Scale-dependent statistical analysis tools can be constructed from the moments of the wavelet coefficients $\widetilde{\boldsymbol{v}}_{\lambda}$ $=\left(\widetilde{v}_{\lambda}^{1}, \widetilde{v}_{\lambda}^{2}, \widetilde{v}_{\lambda}^{3}\right)$ of the vector field $\boldsymbol{v}=\left(v^{1}, v^{2}, v^{3}\right)$. The scale distribution of energy can be computed by summing up the square of the wavelet coefficients at scale $j, \widetilde{E}_{j}$ $=\Sigma_{i_{1}, i_{2}, i_{3}=0}^{j^{j}} \Sigma_{\mu=1}^{7} \Sigma_{l=1}^{3}\left(\widetilde{v}_{\lambda}^{l}\right)^{2} / 2$. The total energy is the sum of the energy per scale; i.e., $E=\Sigma_{j \geqslant 0} \widetilde{E}_{j}$.

The centered $p$ th-order moment of the vector field $\boldsymbol{v}$ at scale $j$ can be defined by

$$
M_{p, j}(\boldsymbol{v})=\frac{1}{3 \times 7 \times 2^{3 j}} \sum_{i_{1}, i_{2}, i_{3}=0}^{2^{j}-1} \sum_{\mu=1}^{7} \sum_{l=1}^{3}\left[\widetilde{v}_{\lambda}^{l}-\bar{M}_{j}(\boldsymbol{v})\right]^{p},
$$

where $\bar{M}_{j}(\boldsymbol{v})=\Sigma_{i_{1}, i_{2}, i_{3}=0}^{j^{j}-1} \Sigma_{\mu=1}^{7} \Sigma_{l=1}^{3} \widetilde{v}_{\lambda}^{l} /\left(3 \times 7 \times 2^{3 j}\right)$ denotes the mean value at scale $j$.

The sparsity of the wavelet coefficients at each scale is a measure of intermittency, and it can be quantified using ratios of moments at different scales

$$
Q_{p, q, j}(\boldsymbol{v})=\frac{M_{p, j}(\boldsymbol{v})}{\left[M_{q, j}(\boldsymbol{v})\right]^{p / q}} .
$$

Classically, one chooses $q=2$ to define typical statistical quantities as a function of scale. For $p=4$, we obtain the scale-dependent flatness $F_{j}=Q_{4,2, j}$. It is equal to 3 at all scales $j$ for a Gaussian white noise, which proves that the signal is not intermittent in this case. For intermittent signals, the flatness increases with $j$. The scale-dependent skewness is obtained for $p=3$. For more details, we refer to Ref. 26 .

\section{Coherent vortex extraction}

We proposed the wavelet-based method coherent vortex extraction (CVE) to extract coherent vortices out of two- and three-dimensional turbulent flows. ${ }^{16,18}$ The CVE method is based on the following principles:

(1) We consider the vorticity field rather than the velocity field, since it preserves Galilean invariance and, moreover, simply connected vortex tubes are preserved by Euler's dynamics (Helmholtz's theorem).

(2) We choose the wavelet representation, where each wave- let coefficient is indexed by both its location and scale, instead of the Fourier representation, where each Fourier coefficient is indexed by its wavenumber but not its location. We prefer to use an orthogonal wavelet basis rather than a biorthogonal one, since only the former yields completely decorrelated coefficients. In particular a Gaussian white noise remains Gaussian and white under orthogonal transformations, while biorthogonal transformations introduce some correlation between the coefficients. $^{20}$

(3) We propose to change our viewpoint concerning coherent structures. Since there has not yet been a universal definition for them, we consider the following minimal but hopefully consensual statement about them: coherent structures are not noise. Using this apophatic method (on the model of negative theology) we then propose the definition: coherent structures correspond to what remains after denoising.

(4) For the noise, we use the mathematical definition stating that a noise cannot be compressed in any functional basis.

(5) We choose, as a first guess, the simplest possible type of noise, namely, additive, Gaussian, and white (uncorrelated) noise.

(6) Since we do not know a priori the variance of the noise, we have developed an iterative procedure ${ }^{27}$ to estimate it from the weakest wavelet coefficients.

(7) Since we consider the case of homogeneous isotropic turbulence, we suppose that each component of the vector field has a similar contribution to the modulus, which is used to compute the variance.

Now we briefly sketch the principle of the CVE algorithm; for more details, we refer the reader to the original papers. ${ }^{16,18}$ The vorticity field $\boldsymbol{\omega}=\boldsymbol{\nabla} \times \boldsymbol{v}$ is first decomposed into an orthogonal wavelet series. We then split the vorticity field into its coherent and incoherent contributions, i.e., $\boldsymbol{\omega}_{\mathbf{c}}(\boldsymbol{x})$ and $\boldsymbol{\omega}_{\mathbf{i}}(x)$, by applying a threshold to the wavelet coefficients. The threshold value is defined as $\widetilde{\varepsilon}$ $=\left(2 / 3 \sigma^{2} \ln N\right)^{1 / 2}$, which depends only on the number of grid points $N$ and on the variance of the incoherent vorticity $\sigma^{2}$ (which is a priori unknown) without any additional adjustable parameters. The choice of this value is based on theorems by Donoho and Johnstone ${ }^{17}$ proving optimality of the wavelet representation to denoise signals in the presence of a Gaussian white noise of variance $\sigma^{2}$, since this waveletbased estimator minimizes the maximal $L^{2}$ error for functions having inhomogeneous regularity. In a first step, we overestimate the variance of the incoherent vorticity by taking the variance of the total vorticity, i.e., $\sigma^{2}=2 Z$, instead. Therewith, we compute the threshold and the variance of all coefficients smaller than this threshold. This yields an improved estimation of the variance of the incoherent vorticity and thus a new threshold can be computed. In Ref. 23, we showed that this procedure can be iterated and that, the stronger the noise level is, the faster the convergence. For the present paper we decided to perform one iteration only to privilege a good compression rate rather than a perfectly denoised contribution. ${ }^{23}$ Finally, the coherent vorticity field $\boldsymbol{\omega}_{\mathbf{c}}$ 
TABLE I. DNS parameters and turbulence characteristics for runs $256^{3}, 512^{3}, 1024^{3}$, and $2048^{3}$. $N$ denotes the number of grid points. $k_{\max } \eta \simeq 1$.

\begin{tabular}{lcccccccc}
\hline \hline & $N$ & $\operatorname{Re}_{\lambda}$ & $k_{\max }$ & $\nu\left(\times 10^{-4}\right)$ & $\langle\epsilon\rangle$ & $L$ & $\lambda$ & $\eta\left(\times 10^{-3}\right)$ \\
\hline Run $256^{3}$ & $256^{3}$ & 167 & 121 & 7.0 & 0.0849 & 1.13 & 0.203 & 7.97 \\
Run $512^{3}$ & $512^{3}$ & 257 & 241 & 2.8 & 0.0902 & 1.02 & 0.125 & 3.95 \\
Run $1024^{3}$ & $1024^{3}$ & 471 & 483 & 1.1 & 0.0683 & 1.28 & 0.090 & 2.10 \\
Run 2048 & $2048^{3}$ & 732 & 965 & 0.44 & 0.0707 & 1.23 & 0.056 & 1.05 \\
\hline \hline
\end{tabular}

is reconstructed from the wavelet coefficients whose moduli are larger than $\widetilde{\varepsilon}$, and the incoherent vorticity field $\boldsymbol{\omega}_{\mathrm{i}}$ from the wavelet coefficients whose moduli are smaller or equal to $\widetilde{\varepsilon}$, or by simple subtraction, i.e., $\boldsymbol{\omega}_{\mathrm{i}}=\boldsymbol{\omega}-\boldsymbol{\omega}_{\mathrm{c}}$, as done in the present paper. The two fields thus obtained, i.e., $\boldsymbol{\omega}_{\mathbf{c}}$ and $\boldsymbol{\omega}_{\mathrm{i}}$, are orthogonal, which insures a separation of the total enstrophy into $Z=Z_{c}+Z_{i}$ because the interaction term $\left\langle\boldsymbol{\omega}_{\mathbf{c}}, \boldsymbol{\omega}_{\mathbf{i}}\right\rangle$ vanishes. We use Biot-Savart's relation $\boldsymbol{v}=-\boldsymbol{\nabla} \times\left(\nabla^{-2} \boldsymbol{\omega}\right)$ to reconstruct the coherent velocity $\boldsymbol{v}_{\boldsymbol{c}}$ and the incoherent velocity $\boldsymbol{v}_{i}$ from the coherent and incoherent vorticities, respectively.

The mathematical properties of the above algorithm have been investigated in Ref. 27 and the influence of the number of iterations has been studied for two-dimensional isotropic turbulence in Ref. 23. For three-dimensional homogeneous isotropic turbulence, the influence is briefly discussed in Appendix B.

There is a large collection of possible orthogonal wavelets and the choice depends on which properties are preferred, e.g., compact-support, symmetry, smoothness, number of cancellations, or computational efficiency. ${ }^{25}$ From our own experience, we prefer the Coifman 12 wavelet, which is compactly supported, has four vanishing moments, is quasisymmetric, and is defined with a filter of length 12 . This leads to computational cost of the fast wavelet transform in $24 N$ operations since two filters are used. We tested different wavelets, e.g., the Coifman wavelets of different order, symlets, spline wavelets, and Haar wavelet. The results obtained by the CVE method are robust to the choice of the wavelet, except for the Haar wavelet which does not have enough cancellations (i.e., only one vanishing moment). A comparison between orthogonal and biorthogonal wavelet decomposition for CVE applied to three-dimensional isotropic turbulence can be found in Ref. 20.

\section{DNS AT $\operatorname{Re}_{\lambda}=167,257,471$, AND 732}

We use the DNS data of three-dimensional incompressible turbulence computed on the ES., ${ }^{3,4}$ The DNS fields obey the Navier-Stokes equations for incompressible fluid with periodic boundary conditions. The simulations use a dealiased pseudospectral method, and a fourth-order RungeKutta method for time marching. The wavenumber increment is 1 and the minimum wavenumber of the DNS is 1 . The total energy $E$ is maintained at an almost timeindependent constant value $(E=0.5)$ by introducing negative viscosity in the wavenumber range $k<2.5$. Readers interested in the DNS may refer to Refs. 3, 4, and 28.

For the readers' convenience, we summarize some key parameters from Ref. 4 in Table I. In Fig. 1, we present four different zooms of isosurfaces showing the intense region where $|\boldsymbol{\omega}|>\langle\omega\rangle+4 \sigma_{\omega}$ for the DNS data at resolution $N$ $=2048^{3}$ and with $\operatorname{Re}_{\lambda}=732$ from Ref. 28 . Here, $\langle\omega\rangle$ is the spatial average of $|\boldsymbol{\omega}|$, and $\sigma_{\omega}$ its standard deviation. Table I presents the parameters of the different simulations of $k_{\max } \eta \simeq 1$, where $k_{\max }$ is the maximum wavenumber of the retained modes, and $\eta$ is the Kolmogorov length scale defined as $\eta=\left(\nu^{3} /\langle\epsilon\rangle\right)^{1 / 4}$. Here $\langle\epsilon\rangle$ is the mean rate of energy dissipation per unit mass. The Taylor-microscale Reynolds number is defined by $\operatorname{Re}_{\lambda}=u^{\prime} \lambda / \nu$, where $\lambda=\left(15 \nu u^{\prime 2} /\langle\epsilon\rangle\right)^{1 / 2}$ is the Taylor microscale, $3 u^{\prime 2} / 2=E$, and $L$ the integral length scale defined as

$$
L=\frac{\pi}{2 u^{\prime 2}} \int_{0}^{k_{\max }} k^{-1} E(k) d k,
$$

where $E(k)$ is the energy spectrum.

\section{COHERENT VORTEX EXTRACTION FOR $\mathrm{Re}_{\lambda}=732$}

Now we apply the coherent vortex extraction algorithm described in Sec. II to the DNS data for $\operatorname{Re}_{\lambda}=732$ computed at $N=2048^{3}$.

\section{A. Total, coherent, and incoherent vorticity}

Figure 2 shows the modulus of vorticity of the total flow (green) for the case $\operatorname{Re}_{\lambda}=732$, after zooming on a $256^{3}$ subcube to enhance structural details. The flow exhibits elongated, distorted, and folded vortex tubes, as observed in laboratory ${ }^{10}$ and numerical ${ }^{6-9}$ experiments. We then decompose the flow into its coherent and incoherent contributions and plot isosurfaces of the coherent (red) and incoherent (blue) vorticity for $|\boldsymbol{\omega}|=5 \sigma$, and $5 \sigma / 3$, respectively, with the root mean square $\sigma=(2 Z)^{1 / 2}$. We observe that the coherent vorticity, represented by $2.6 \% \mathrm{~N}$ wavelet coefficients, retains $99.8 \%$ of the energy and $79.8 \%$ of the enstrophy. Moreover, the coherent vorticity exhibits the same vortex tubes as those present in the total vorticity; we have checked that both fields plotted with the same isosurfaces $|\boldsymbol{\omega}|=5 \sigma$ well superimpose. In contrast, the incoherent vorticity is structureless; we have checked this by zooming in that there are no vortex tubes left. Note that the value of the isosurface chosen for visualization is the same for the total and coherent vorticity, but it has been reduced by a factor of 3 for the incoherent vorticity, whose fluctuations are much smaller. 

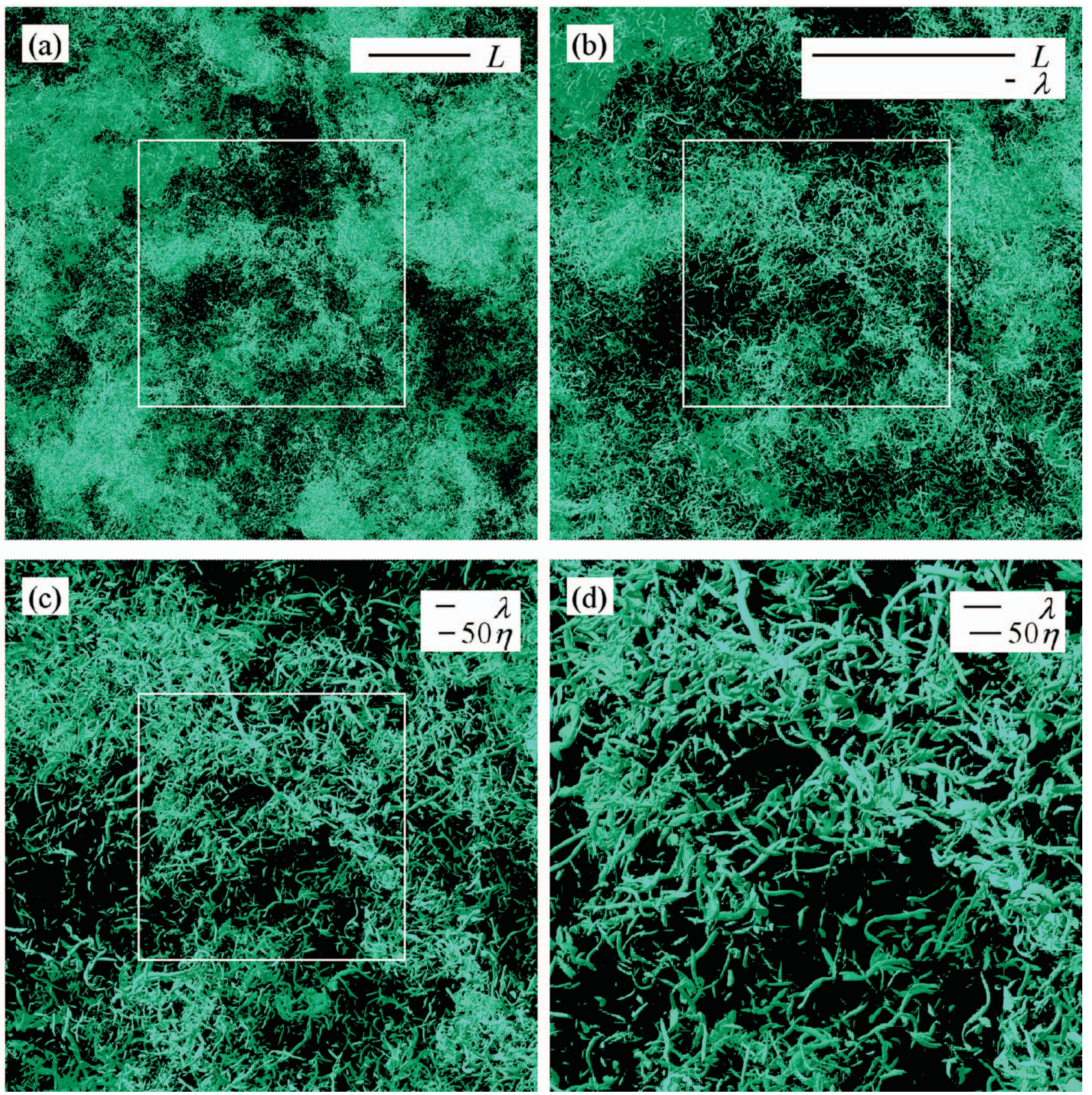

FIG. 1. (Color) Different zooms of isosurfaces of vorticity for $\operatorname{Re}_{\lambda}=732$ at resolution $N=2048^{3}$ (from Ref. 28). Isosurfaces of vorticity for $|\boldsymbol{\omega}|>\langle\omega\rangle+4 \sigma_{\omega}$. (a) The size of the display domain is $\left(5984^{2} \times 1496\right) \eta^{3}$, periodic in the vertical and horizontal directions. (b) Close-up view of the central region of (a) bounded by the white rectangular line; size of the display domain is $\left(2992^{2} \times 1496\right) \eta^{3}$. (c) Close-up view of the central region of (b): $1496^{3} \eta^{3}$. (d) Close-up view of the central region of $(\mathrm{c}):\left(748^{2} \times 1496\right) \eta^{3}$.

\section{B. Velocity and vorticity probability density functions (PDFs)}

The PDFs of velocity and vorticity of the total, coherent, and incoherent flows estimated by histograms using 200 bins are depicted in Fig. 3. The comparison of the total and coherent velocity PDFs (two wide PDFs in the top figure) shows that they coincide well. The two narrow PDFs show that the incoherent velocity PDF is quasi-Gaussian with a strongly reduced variance compared to that of the total velocity. Likewise the PDFs of the total and coherent vorticity almost superimpose and show a stretched exponential behavior which reflects the flow intermittency due to the presence of coherent vortices. The PDF of the incoherent vorticity has an exponential shape with a reduced variance compared to that of the total vorticity.

\section{Energy spectra}

For the case $\operatorname{Re}_{\lambda}=732$, Fig. 4 shows that the spectrum of the coherent energy is identical to that of the total energy all along the inertial range. This implies that coherent vortices are responsible for the $k^{-5 / 3-0.1}$ energy scaling shown in Ref. 4. In the dissipative range, i.e., for $k \eta \gtrsim 0.3$, the spectrum of the coherent energy differs from that of the total energy, 

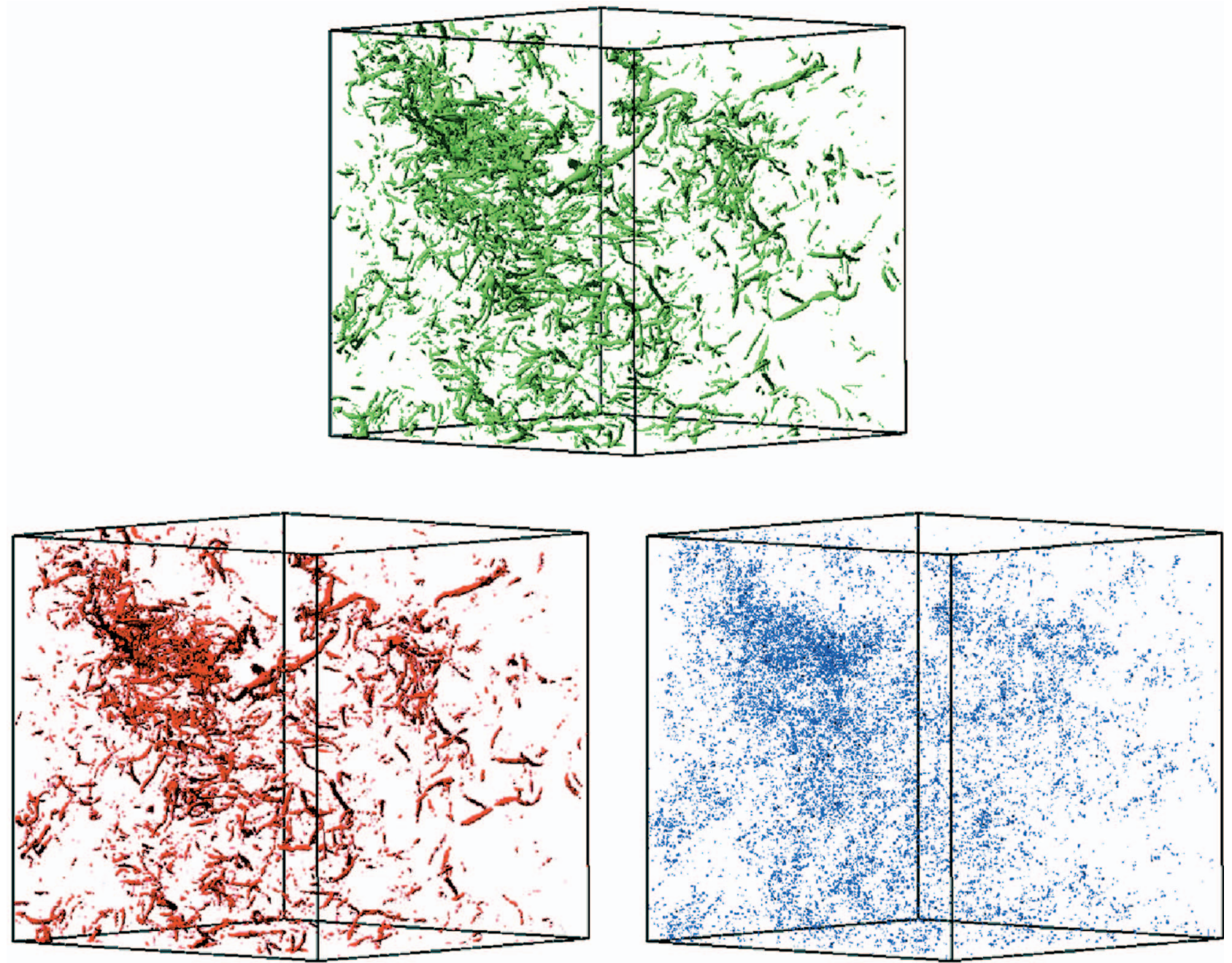

FIG. 2. (Color) Isosurfaces of total (top), coherent (bottom left), and incoherent (bottom right) vorticity for $\operatorname{Re}_{\lambda}=732$ at resolution $N=2048^{3}$. The values of the isosurfaces are $|\boldsymbol{\omega}|=5 \sigma$ for the total and coherent vorticities, and only $|\boldsymbol{\omega}|=(5 / 3) \sigma$ for the incoherent vorticity. Only subcubes of size $256^{3}$ are visualized, although computation has been performed at resolution $N=2048^{3}$.

although there is still a significant contribution of the coherent vortices at scales smaller than $k \eta \simeq 0.3$. Concerning the incoherent flow, we observe that the scaling of the incoherent energy spectrum is close to $k^{2}$, which corresponds to an equipartition of incoherent energy between all wavenumbers $\boldsymbol{k}$, since the isotropic spectrum is obtained by integrating energy in 3D $\boldsymbol{k}$-space over spherical shells $k=|\boldsymbol{k}|$. The incoherent velocity is therefore spatially decorrelated, which is consistent with the observation that incoherent vorticity is structureless (Fig. 2). We also observe for the total flow that there is some energy piling up around $k \eta \simeq 1$, which corresponds to the cutoff wavenumber. This spike is retained by the incoherent contribution but not by the coherent contribution.

\section{Velocity skewness and flatness}

The scale-dependent skewness and flatness for the total, coherent, and incoherent velocities at $\operatorname{Re}_{\lambda}=732$ are shown in Figs. 5 and 6 as a function of the dimensionless wavenumber $k_{j} \eta$, respectively. Here, $k_{j}=2^{j} / 1.3$ where $1 / 1.3$ is the centroid wavenumber of the Coifman 12. In both figures, we only show the five smallest scales. At low wavenumbers, the statistical quantities of the incoherent contribution yield erroneous results, since too few wavelet coefficients of the incoherent vorticity field represent its contribution at large scales. Therefore they have been omitted. The skewness shown in Fig. 5 tends to zero for the incoherent flow as the wavenumber increases, while those of the coherent and total flow become slightly negative. Figure 6 shows that the flatness of the total and coherent flow increases with the wavenumber. For the coherent flow we even find a stronger increase than for the total one, which illustrates that the coherent vortices are responsible for the flow intermittency. The flatness of the incoherent flow has much smaller values, but are not equal to 3, the value for a Gaussian noise.

\section{E. Energy transfers and fluxes}

Studying the energy transfer in Fourier space enables us to check the contributions of the coherent and incoherent flows to the nonlinear dynamics. For this the total, coherent and incoherent velocity fields are transformed into Fourier space: 

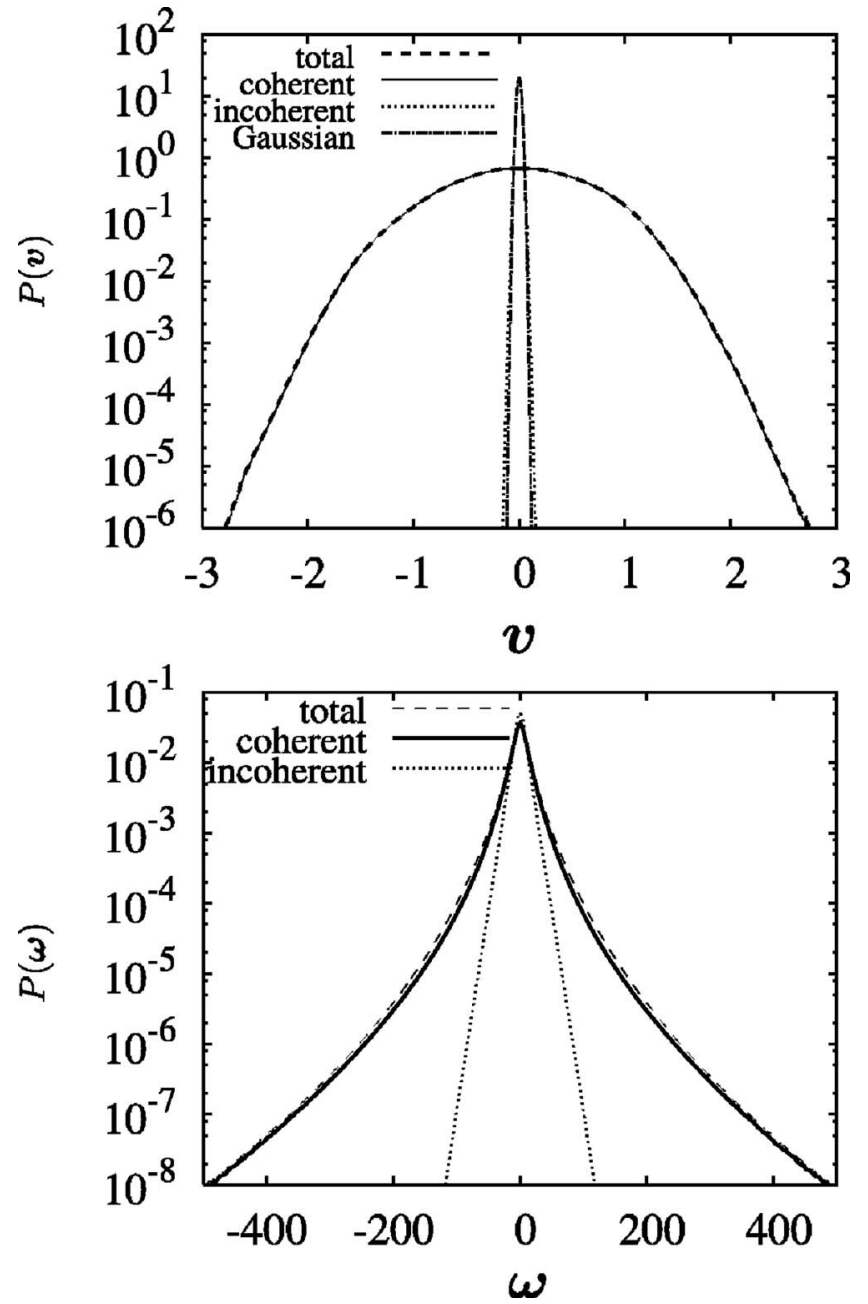

FIG. 3. PDFs of velocity (top) and vorticity (bottom) for the case $\operatorname{Re}_{\lambda}$ $=732$ at resolution $N=2048^{3}$. The Gaussian distribution in the top figure is normalized so that it has zero mean and the same standard deviation as that of the incoherent velocity.

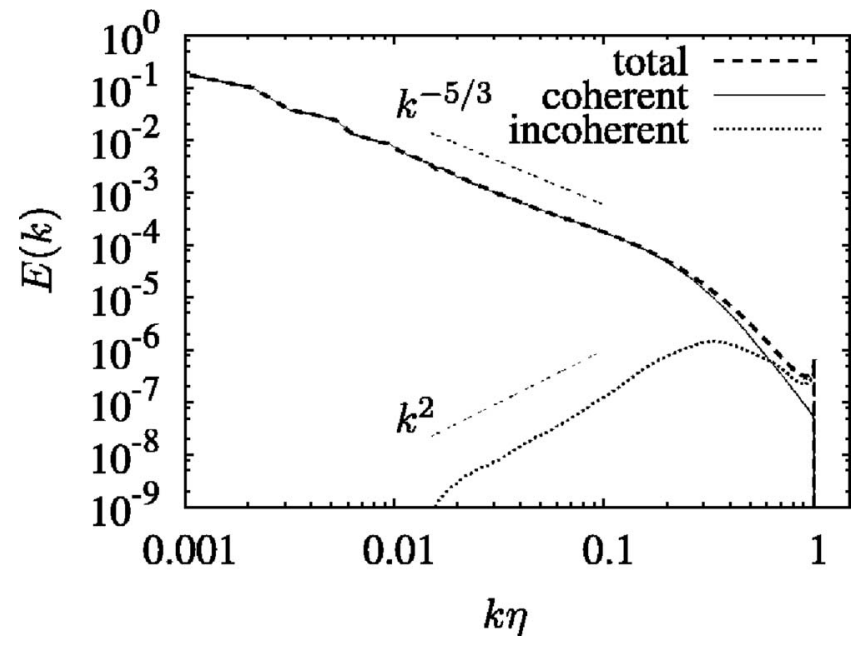

FIG. 4. Energy spectra of the total, coherent, and incoherent flow for the case $\operatorname{Re}_{\lambda}=732$ computed at resolution $N=2048^{3}$.

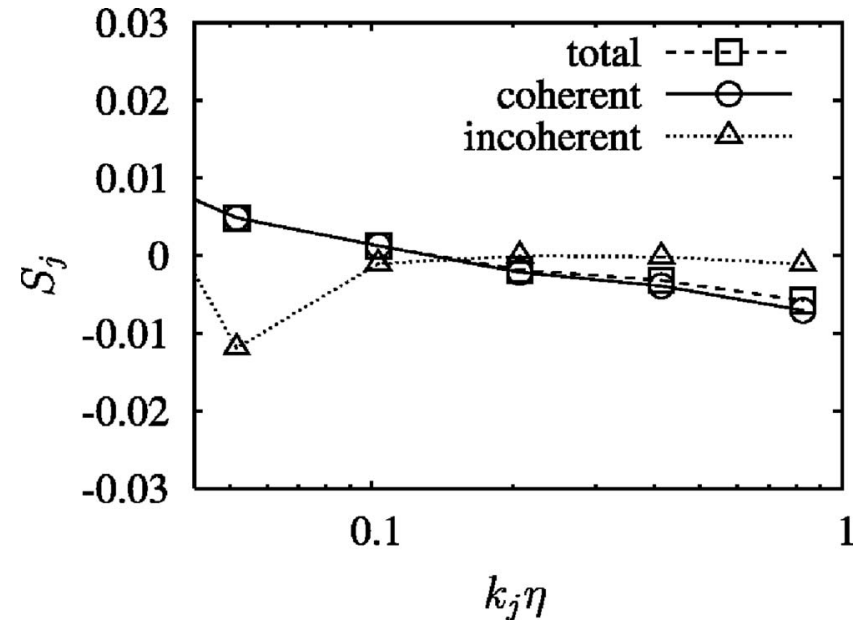

FIG. 5. Scale-dependent skewness of velocity vs wavenumber for $\operatorname{Re}_{\lambda}$ $=732$.

$$
\mathcal{F}[\boldsymbol{v}](\boldsymbol{k})=\int_{\mathbf{R}^{3}} \boldsymbol{v}(\boldsymbol{x}) \exp (-\mathrm{i} 2 \pi \boldsymbol{k} \cdot \boldsymbol{x}) d \boldsymbol{x} .
$$

The energy transfer function $T(k)$ of the total flow is then defined by

$$
T(k)=\sum_{k-1 / 2 \leqslant|\boldsymbol{p}|<k+1 / 2} \mathcal{F}[\boldsymbol{v}](-\boldsymbol{p}) \cdot \mathcal{F}[(\boldsymbol{v} \cdot \boldsymbol{\nabla}) \boldsymbol{v}](\boldsymbol{p}),
$$

where we sum over spherical shells in $k$-space, and the corresponding energy flux $\Pi(k)$ is defined by

$$
\Pi(k)=-\int_{0}^{k} T(k) d k .
$$

According to the above formulae, the energy fluxes of the different velocity contributions can be computed. Using the decomposition $\boldsymbol{v}=\boldsymbol{v}_{c}+\boldsymbol{v}_{i}$, we obtain eight transfer terms for all possible combinations between coherent and incoherent contributions. We introduce

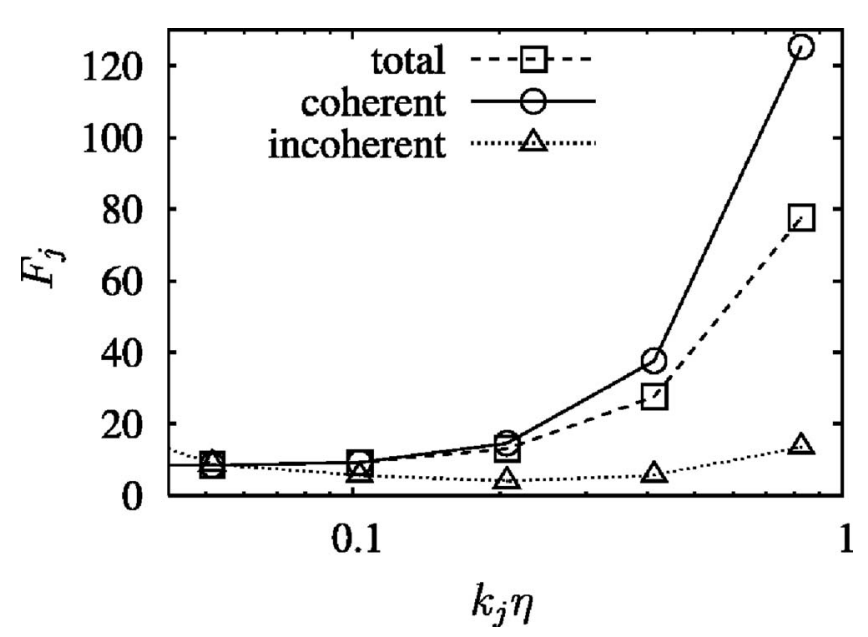

FIG. 6. Scale-dependent flatness of velocity vs wavenumber for $\mathrm{Re}_{\lambda}=732$. 


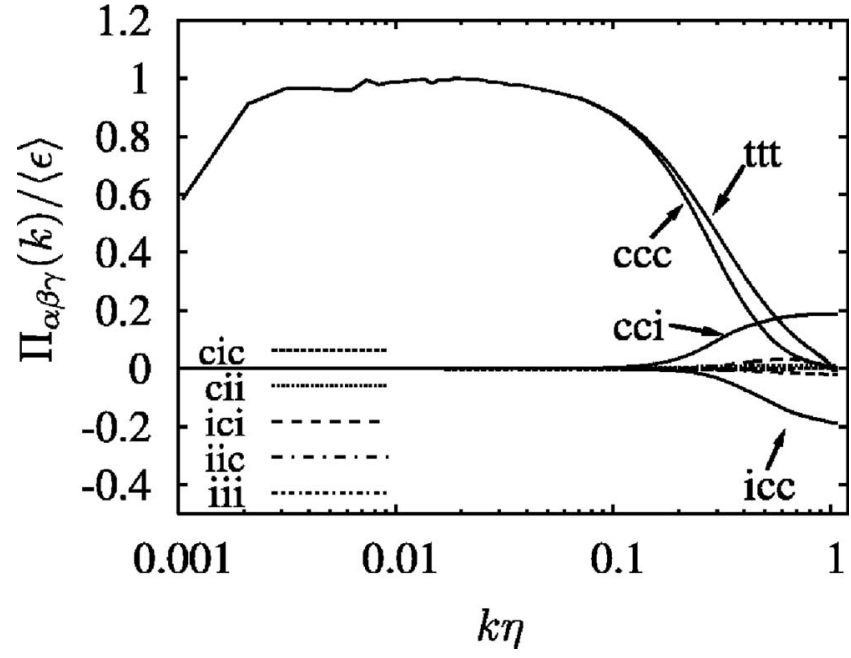

FIG. 7. Energy fluxes $\boldsymbol{\Pi}_{\alpha \beta \gamma}(k) /\langle\epsilon\rangle$ vs wavenumber of the different flow contributions for the case $\operatorname{Re}_{\lambda}=732$.

$$
T_{\alpha \beta \gamma}(k)=\sum_{k-1 / 2 \leqslant|\boldsymbol{p}|<k+1 / 2} \mathcal{F}\left[\boldsymbol{v}_{\alpha}\right](-\boldsymbol{p}) \cdot \mathcal{F}\left[\left(\boldsymbol{v}_{\beta} \cdot \boldsymbol{\nabla}\right) \boldsymbol{v}_{\gamma}\right](\boldsymbol{p}),
$$

and obtain the corresponding energy flux; i.e., $\Pi_{\alpha \beta \gamma}(k)$ $=-\int_{0}^{k} T_{\alpha \beta \gamma}(k) d k$, for $(\alpha, \beta, \gamma) \in\{\mathrm{c}, \mathrm{i}\}$. Thus, we can define the eight fluxes: $\Pi_{\mathrm{ccc}}, \Pi_{\mathrm{cci}}, \Pi_{\mathrm{cic}}, \Pi_{\mathrm{cii}}, \Pi_{\mathrm{icc}}, \Pi_{\mathrm{ici}}, \Pi_{\mathrm{iic}}$, and $\Pi_{\mathrm{iii}}$.

In Fig. 7 we plot the eight energy fluxes normalized by the dissipation rate $\Pi(k) /\langle\epsilon\rangle$ vs $k \eta$, together with the total flux denoted by $\Pi_{\mathrm{ttt}}$. As expected, we find that $\Pi_{\mathrm{ccc}}$ coincides with $\Pi_{\mathrm{ttt}}$ all along the inertial range, which confirms that the nonlinear dynamics is fully captured by the coherent flow. All along the inertial range the other fluxes are almost zero. In the dissipative range we observe that the coherent flux $\Pi_{\mathrm{ccc}}$ still dominates, though it begins to depart from the total flux $\Pi_{\mathrm{ttt}}$, since $\Pi_{\mathrm{cci}}$, and $\Pi_{\mathrm{icc}}$ begin to build up for scales smaller than $k \eta \simeq 0.1$. The flux $\Pi_{\text {cci }}$ is positive while the flux $\Pi_{\text {icc }}$ is negative and they tend to compensate each other since they have almost the same magnitude for $k \eta \rightarrow 1$. We also find that $\Pi_{\text {cci }}$ and $\Pi_{\text {icc }}$ become more important for $k \eta \rightarrow 1$. The remaining terms are negligible.

\section{INFLUENCE OF THE REYNOLDS NUMBER FROM $\operatorname{Re}_{\lambda}=167$ TO 732}

We apply the coherent vortex extraction algorithm to four different DNS datasets to study the influence of the Reynolds number from $\operatorname{Re}_{\lambda}=167$ to 732 .

\section{A. Compression rate}

The compression rate $C$ is defined as $C=100 N_{c} / N$, where $N_{c}$ denotes the number of retained coefficients and $N$ the total number of coefficients. The compression rate corresponds to the percentage of coherent coefficients that are kept. We observe in Fig. 8 (top) that $C$ decreases from $3.6 \%$ for $\operatorname{Re}_{\lambda}=167$ to $2.6 \%$ for $\operatorname{Re}_{\lambda}=732$, according to $C$ $\propto \operatorname{Re}_{\lambda}^{-0.21}$. The exponents in Figs. 8 and 9 are estimated by a least-squares fit of the four available data points. This $\operatorname{Re}_{\lambda}$ dependence shows that the flow intermittency increases with
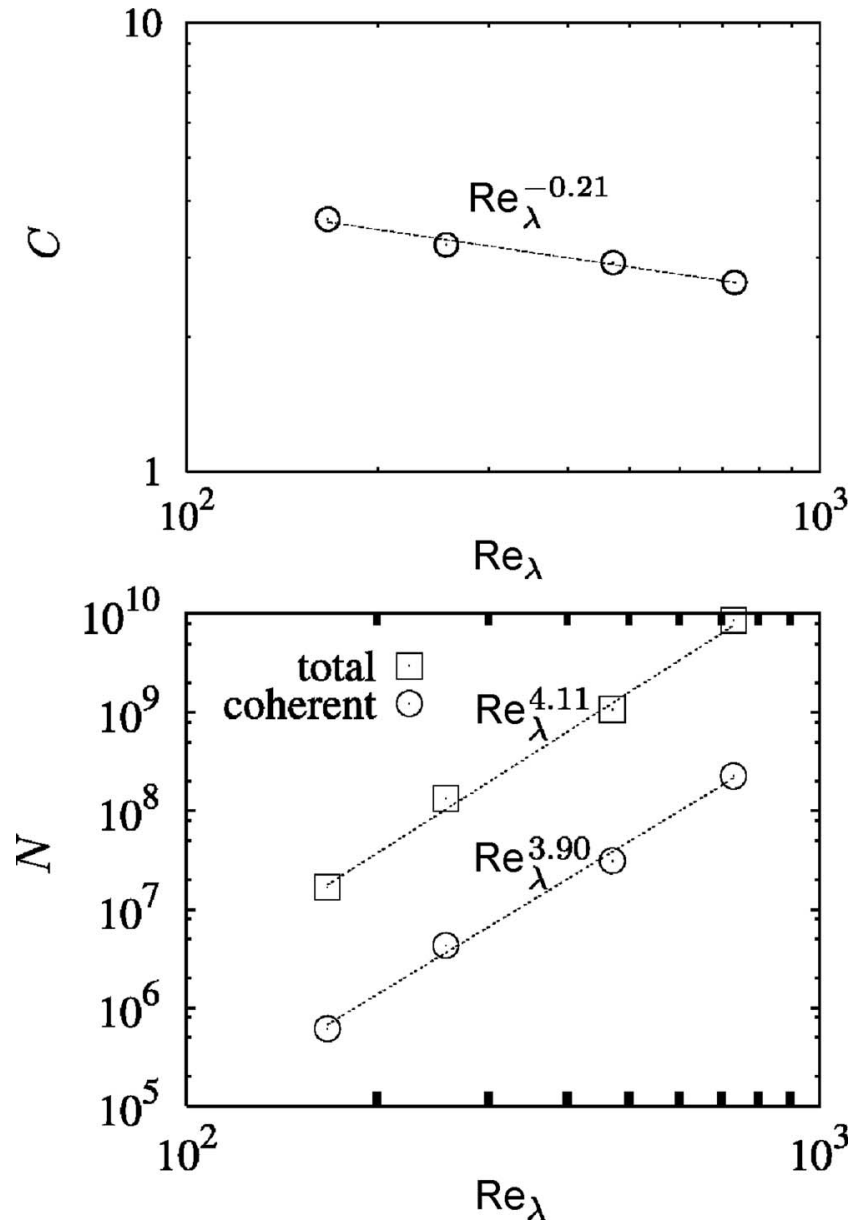

FIG. 8. Compression rate (top) and number of coefficients (bottom) vs $\operatorname{Re}_{\lambda}$.

$\mathrm{Re}_{\lambda}$, which is consistent with the previous experimental results presented in Fig. 6 of Ref. 29. From this observation, we conjecture that the higher the Reynolds number, the more efficient the wavelet representation is.

Figure 8 (bottom) depicts the number of retained coefficients for the total and the coherent parts versus the Reynolds number. As the compression rate decreases with the Reynolds number, we observe that the number of coefficients of the coherent part grows more slowly than that of the total flow. For the case $\operatorname{Re}_{\lambda}=732$, the coherent part corresponds to about $2.3 \times 10^{8}$ coefficients, while the total flow corresponds to about $8.6 \times 10^{9}$ coefficients. The number $N$ of modes in the DNS presented here (computed up to resolution $2048^{3}$ ) increases with $\operatorname{Re}_{\lambda}^{4.11}$. Kolmogorov's theory predicts the scaling $N \propto \operatorname{Re}_{\lambda}^{9 / 2}$, which is confirmed in Ref. 28 using one more data point, corresponding to a DNS at resolution $4096^{3}$. For the number of coherent modes, we find the $\operatorname{Re}_{\lambda}$ dependence, i.e., $N_{\mathrm{c}} \propto \operatorname{Re}_{\lambda}^{3.90}$, which shows the number of degrees of freedom increases more slowly than the one for DNS.

Figure 9 shows energy and enstrophy versus $\operatorname{Re}_{\lambda}$ for the total, coherent, and incoherent flows. The energy of the total and coherent flows coincide (Fig. 9, top). The energy of the total flow is maintained at an almost time-independent constant value $(E=0.5)$ by introducing negative viscosity in the DNS data we analyzed (see Sec. III). We also find that the energy of the incoherent flow decreases with $\operatorname{Re}_{\lambda}$ approxi- 

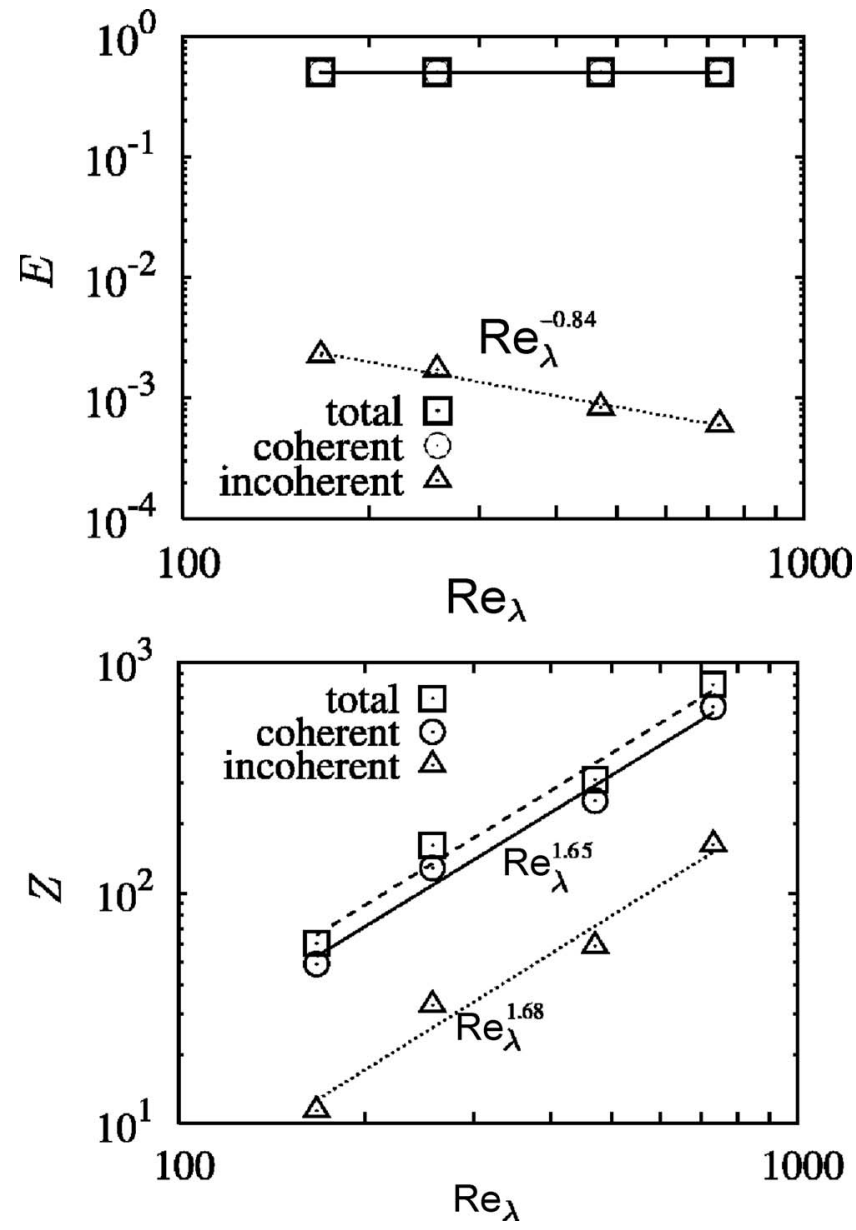

FIG. 9. Energy (top) and enstrophy (bottom) for the total, coherent, and incoherent flow vs $\operatorname{Re}_{\lambda}$.

mately as $E_{i} \propto \operatorname{Re}_{\lambda}^{-0.84}$. For the enstrophy (Fig. 9, bottom) we find that both, the total and the coherent enstrophy, increase; i.e., $Z_{\mathrm{t}} \propto \operatorname{Re}_{\lambda}^{1.65}$ and $Z_{c} \propto \operatorname{Re}_{\lambda}^{1.65}$. We also observe that the incoherent enstrophy is increasing with $Z_{i} \propto \operatorname{Re}_{\lambda}^{1.68}$. The $\operatorname{Re}_{\lambda}$ dependence of the different enstrophies are all very similar.

The energy and enstrophy of the coherent/incoherent flows are listed in Table II.

\section{B. Scale-dependent compression rate}

Figure 10 shows the scale-dependent compression rate $C_{j}$ defined by $C_{j}=100 N_{c, j} / N_{j}$; i.e., the percentage of coefficients corresponding to the retained coherent part at scale $j$, plotted versus the normalized wavenumber $k_{j} \eta$ for the different cases. Here, $N_{c, j}$ is the number of the retained wavelet coefficients at scale $j, N_{j}=7 \times 2^{3 j}$ the total number of coeffi-

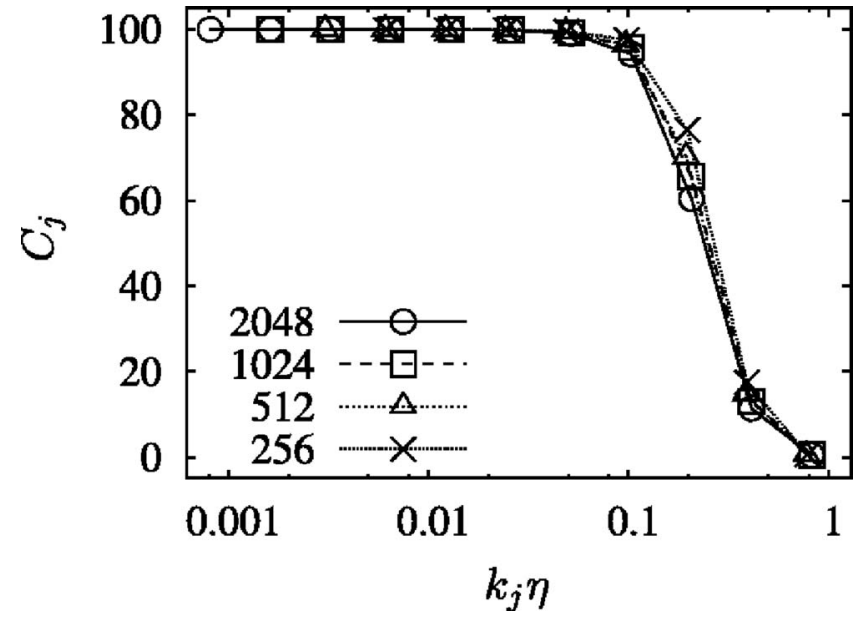

FIG. 10. Scale-dependent compression rate $C_{j}$ vs $k_{j} \eta$ for runs $256^{3}, 512^{3}$, $1024^{3}$, and $2048^{3}$.

cients at scale $j$, and $k_{j}$ is the centroid wavenumber of the Coifman 12 wavelet. For all Reynolds numbers, we observe that the compression rate $C_{j}$ decreases with increasing $k_{j} \eta$. For $k_{j} \eta \lesssim 0.1$, almost all coefficients are retained by the coherent part, while the percentage of the retained coefficients decreases for $k_{j} \eta \geq 0.1$; i.e., the compression rate is improved. Note that due to the octree representation of the wavelet coefficients, the number $N_{j}$ is very large for small scales (i.e., for large $j$ ), so that the overall compression rate $C$ is dominated by $C_{j}$ at small scales. We also observe that, for increasing values of $\mathrm{Re}_{\lambda}$, the compression rate per scale decreases faster as scale becomes smaller; i.e., the rate is reduced at larger $\operatorname{Re}_{\lambda}$ for $k_{j} \eta \gtrsim 0.1$. This reflects that the larger the Reynolds number, the better the wavelet-based extraction.

\section{Energy spectra}

The compensated energy spectra $\left[k^{5 / 3} E(k) /\langle\epsilon)^{2 / 3}\right]$ plotted versus the normalized wavenumber $k \eta$ illustrate that the slope of the inertial range is -0.1 below the Kolmogorov slope of $-5 / 3$ for both, the total (Fig. 11, upper curves) and coherent flows (Fig. 11, lower curves). The shoulders of the energy spectrum with a maximum around $k \eta \sim 0.13$ are also well retained in the coherent flows.

However, in the dissipative range, we also observe that the spectra of the coherent flow decay faster than that of the total flow and that the energy does not pile up.

The compensated energy spectra $\left[E(k) /\left(k^{2} \eta^{11 / 3}\langle\epsilon\rangle^{2 / 3}\right)\right]$ of the incoherent flows, plotted in Fig. 12, show that the

TABLE II. The percentages and absolute values of coherent/incoherent energy and enstropy for runs $256^{3}, 512^{3}$, $1024^{3}$, and $2048^{3}$.

\begin{tabular}{lcccccccc}
\hline \hline & $E_{c}[\%]$ & $E_{i}[\%]$ & $E_{c}$ & $E_{i}$ & $Z_{c}[\%]$ & $Z_{i}[\%]$ & $Z_{c}$ & $Z_{i}$ \\
\hline Run 256 & 99.1 & 0.45 & 0.4955 & 0.0023 & 81.2 & 18.8 & 49.3 & 11.4 \\
Run 512 & 99.3 & 0.34 & 0.4966 & 0.0017 & 79.7 & 20.3 & 128.3 & 32.7 \\
Run $1024^{3}$ & 99.7 & 0.17 & 0.4983 & 0.0008 & 81.0 & 19.0 & 251.5 & 59.0 \\
Run 2048 & 99.8 & 0.14 & 0.4988 & 0.0007 & 79.8 & 20.2 & 641.0 & 162.3 \\
\hline \hline
\end{tabular}




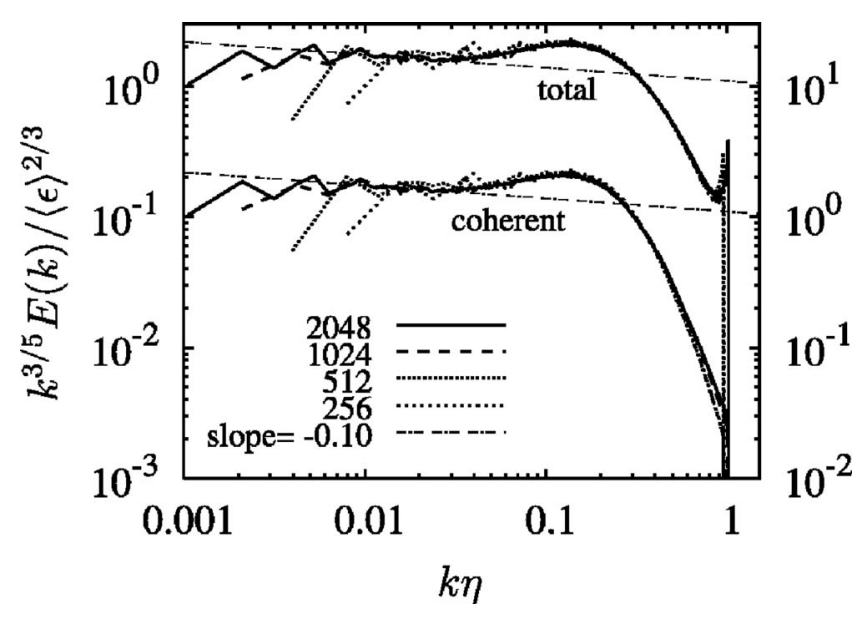

FIG. 11. Compensated energy spectra of the total and coherent flows for runs $256^{3}, 512^{3}, 1024^{3}$, and $2048^{3}$. Scales on the left and right are for the total and the coherent flows, respectively.

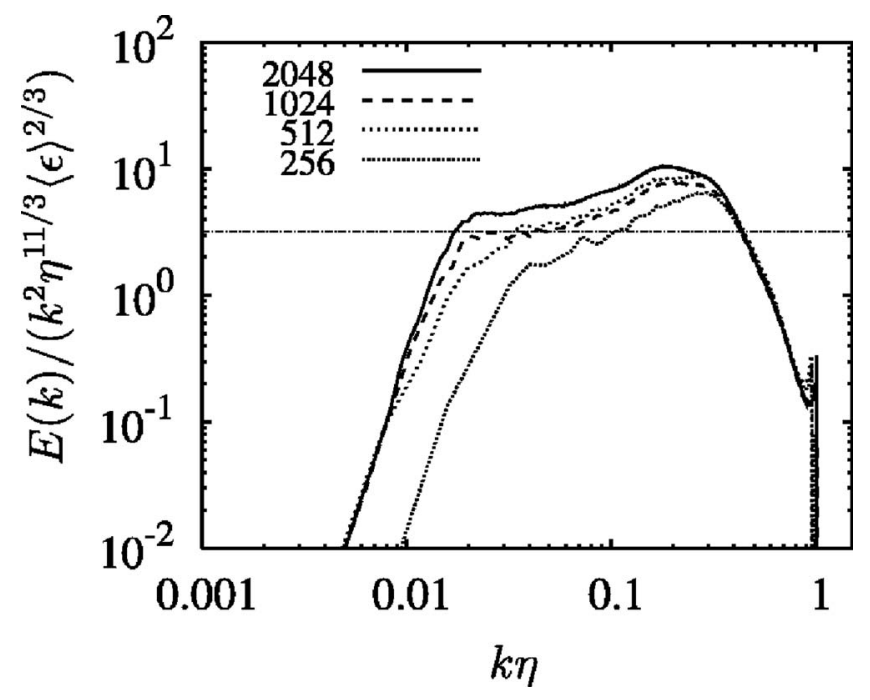

FIG. 12. Compensated energy spectra of the incoherent flows for runs $256^{3}$, $512^{3}, 1024^{3}$, and $2048^{3}$.

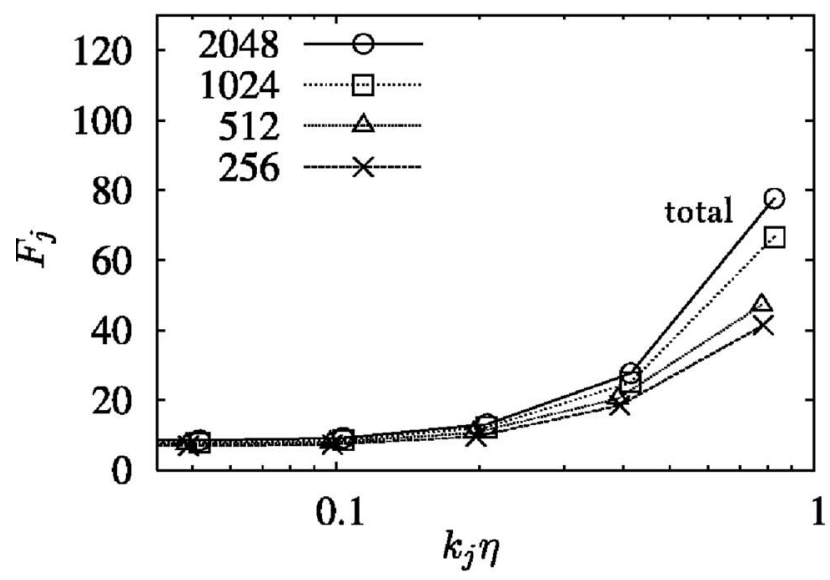

range of a $k^{2}$ slope is increasing with $\mathrm{Re}_{\lambda}$. The shoulders, similar to that observed in the spectra of the total and the coherent flows, are also present in the spectra of the incoherent flows.

\section{Velocity flatness}

The scale-dependent flatness for the total, coherent and incoherent velocity from $\operatorname{Re}_{\lambda}=167$ to 732 is shown in Fig. 13. As done in Sec. IV D, we only plot the five smallest scales. We observe that the flatness of the total and coherent flows increases with $\operatorname{Re}_{\lambda}$ for each scale, while the flatness of the incoherent flows is almost independent of $\mathrm{Re}_{\lambda}$. The increase for the total flows with $\operatorname{Re}_{\lambda}$ at each scale leads to an improvement of the scale-dependent compression rate $C_{j}$ when increasing $\operatorname{Re}_{\lambda}$ (Fig. 10). We also find that the flatness of the total and coherent flows increases with $k_{j} \eta$ for all $\operatorname{Re}_{\lambda}$, which is consistent with the improvement of $C_{j}$ as $k_{j} \eta$ increases. At each $\operatorname{Re}_{\lambda}$, we find that the flatness increases faster with $k_{j} \eta$ for the coherent flow than for the total flow.

\section{CONCLUSION AND PERSPECTIVES}

We have introduced a wavelet-based denoising method that splits each flow realization into two orthogonal components-one coherent and organized, and the other incoherent and random-both being active all along the inertial range. We have proposed this as a way to circumvent the fact that there is no spectral gap to facilitate the modeling of turbulence, in contrast to other situations where classical statistical physics can be successfully used (e.g., solid state physics). Another advantage of this method, called CVE (coherent vortex extraction), is to propose a constructive definition of coherent structures which is based on the minimalist assumption that coherent structures are not noise. It therefore does not require a template of the shape or any more precise definition of coherent structures.

Here we applied the CVE method to DNS data of statistically stationary homogeneous isotropic turbulent flows forced at large scales and computed at resolutions $256^{3}, 512^{3}, 1024^{3}$, and $2048^{3}$, corresponding to Taylor microscale Reynolds numbers of $\operatorname{Re}_{\lambda}=167,257$, 471, and 732, respectively. The flow fields are characterized by a large

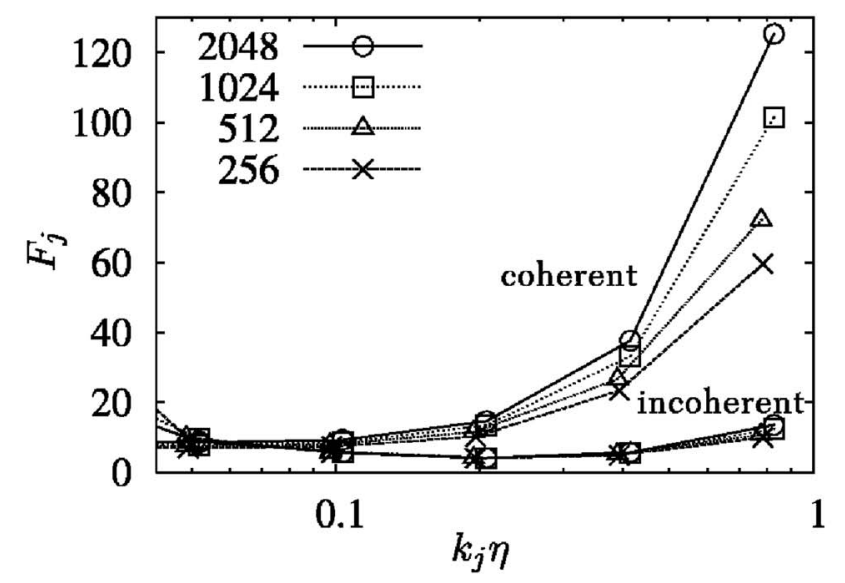

FIG. 13. Scale-dependent flatness of (left) total, (right) coherent, and incoherent velocity for runs $N=256^{3}, 512^{3}, 1024^{3}$, and $2048^{3}$. 
range of active scales, which increases with the Reynolds number. The wavelet representation detects the flow intermittency, which is characterized by the fact that only few coefficients have significant values in the small scales.

We have shown that few wavelet coefficients are sufficient to represent the coherent vortices, while the large majority of the coefficients corresponds to an incoherent background flow, which is structureless and contains no vortex tubes. The statistics of the coherent flow is similar to that of the total flow since the coherent flow contains most of the energy and enstrophy of the total flow. Their energy spectrum coincides all along the inertial range and differs only in the dissipative range. The velocity and vorticity PDFs are in good agreement with those of the total flow. In contrast, the statistics of the incoherent flow, which contains much less energy and enstrophy, differ: the energy tends to be equidistributed among the incoherent modes, while the velocity PDF is quasi-Gaussian with a strongly reduced variance. These results confirm for much higher Reynolds numbers, at least up to $\operatorname{Re}_{\lambda}=732$, the conclusions we obtained for lower Reynolds numbers $\operatorname{Re}_{\lambda}=150$ and $168{ }^{18,30}$

Checking the influence of the Reynolds number, we confirmed that the above results hold for all Reynolds numbers we investigated and that the percentage of wavelet coefficients corresponding to the coherent vortices decreases from $3.6 \%$ to $2.6 \%$ as $\operatorname{Re}_{\lambda}$ increases from 167 to 732 . The improvement of the compression rate implies that the $\mathrm{Re}_{\lambda}$ dependence of $N_{c}$, which is the number of degrees of freedom necessary to represent the coherent flow, is weaker than the estimation based on the statistical theory of Kolmogorov which is typically used for DNS, i.e., $N \propto \operatorname{Re}_{\lambda}^{9 / 2}$, where $N$ is the number of the degrees of freedom in DNS. The flow intermittency increases with the Reynolds number at least up to $\operatorname{Re}_{\lambda}=732$, since the wavelet representation of the coherent vortices becomes sparser, and hence more efficient, as the Reynolds number increases. For the four Reynolds numbers considered here, by the use of a least-square method, we found that $N_{c}$ increases with $\operatorname{Re}_{\lambda}$ approximately as $N_{c}$ $\propto \operatorname{Re}_{\lambda}^{3.9}$.

The analyses of the nonlinear dynamics we have made by computing the energy transfer and flux of the different flow contributions in terms of the wavenumber have shown that the coherent contribution captures the nonlinear dynamics all along the inertial range. In contrast, the incoherent contribution becomes non-negligible only in the dissipative range.

The above results motivate further developments of the coherent vortex simulation (CVS) method. It is based on a deterministic computation of the time evolution of the coherent flow using an adaptive wavelet basis, while the influence of the incoherent flow onto the coherent flow is neglected or statistically modeled. First results of CVS for a threedimensional turbulent mixing layer are promising, ${ }^{23}$ and the new estimation presented in this paper shows that it might become more efficient as the Reynolds number increases, since the percentage of retained coherent modes decreases.

\section{ACKNOWLEDGMENTS}

The computations were carried out on HPC2500 system at the Information Technology Center of Nagoya University. This work was supported by the Grant-in-Aid for the 21st Century COE "Frontiers of Computational Science" and also by Grant-in-Aid for Scientific Research No. (B) 17340117 from the Japan Society for the Promotion of Science. M.F. and K.S. acknowledge financial support from IPSL, Paris and from Nagoya University. N.O. and K.Y. also thank Ecole Normale Supérieure for hospitality during their stay in Paris. The authors would like to express their thanks to Takashi Ishihara, Mitsuo Yokokawa, Ken'ichi Itakura, and Atsuya Uno for providing us with the DNS data. They thank Takashi Ishihara and Tomohiro Aoyama for their support of the DNS data handling and for providing a fast Fourier transform code that they parallelized by the use of MPI library in collaboration with Masaaki Osada, and also thank Giulio Pellegrino for providing his serial wavelet decomposition code, Margarete Domingues and Thierry Goldmann for helping to visualize Fig. 2 using IDRIS Super Computing Center in Orsay.

\section{APPENDIX A: DIVERGENCE PROBLEM}

As the orthogonal wavelet transform does not commute with the divergence operator and the vector-valued wavelet basis is not divergence-free, the coherent vortex extraction does not yield coherent and incoherent vorticity fields that are divergence-free. However, this is not a key issue in practice, since the divergent component of the decomposed vorticity field remains less than $2.17 \%$ of the total enstrophy (for $\operatorname{Re}_{\lambda}=732$ ) and appears mostly in the dissipative range as shown in the following.

In Fig. 14 we plot the enstrophy spectra of the coherent and incoherent vorticities together with the divergence-free counterparts, denoted by $\boldsymbol{\omega}_{\mathbf{c}}^{\prime}, \boldsymbol{\omega}_{\mathrm{i}}^{\prime}$, and the divergent part $\boldsymbol{\nabla} \phi$. We observe that the divergent part actually contributes little to the enstrophy, and this only in the dissipative range. This is even less than what we have already observed for $\operatorname{Re}_{\lambda}$ $=150$ computed at $N=256^{3} .{ }^{30}$ Therefore, we think that this lack of having not perfectly divergence-free vorticity fields does not pose a problem for CVS.

\section{APPENDIX B: INFLUENCE OF THE NUMBER OF ITERATIONS IN THE CVE METHOD}

We briefly discuss how the compression rate $C$ is influenced by the number of iterations $\mathcal{I}$ in the CVE algorithm. Figure 15 shows that compression rate $C$ monotonically increases with $\mathcal{I}$ and converges after about ten iterations whatever $\mathrm{Re}_{\lambda}$. Moreover, $C$ decreases when $\mathrm{Re}_{\lambda}$ increases, irrespective of $\mathcal{I}$, e.g., $C=8.7 \%$ for $\operatorname{Re}_{\lambda}=167$, and only $6.0 \%$ for $\operatorname{Re}_{\lambda}=732$. The $\operatorname{Re}_{\lambda}$ dependence of $C$ after convergence (Fig. 16) is similar to what we have obtained for one iteration only (Fig. 8, top). Figures 17 and 18 show that the statistics are similar to those obtained using one iteration only (compare Figs. 3 and 4). 


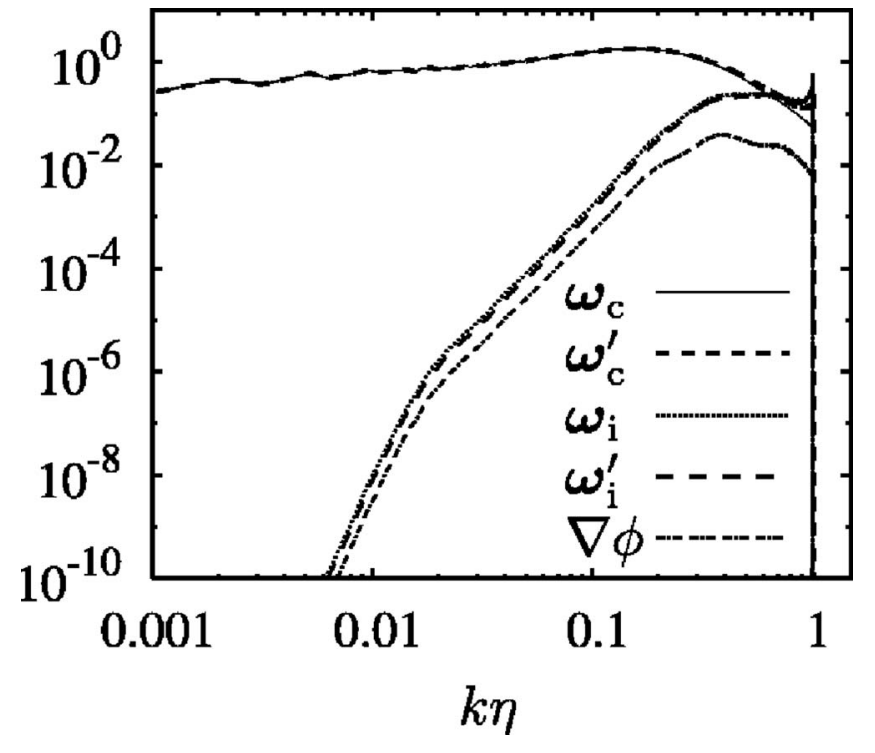

FIG. 14. Enstrophy spectra of the divergence-free contribution for the case $\operatorname{Re}_{\lambda}=732$ and $N=2048^{3}$.

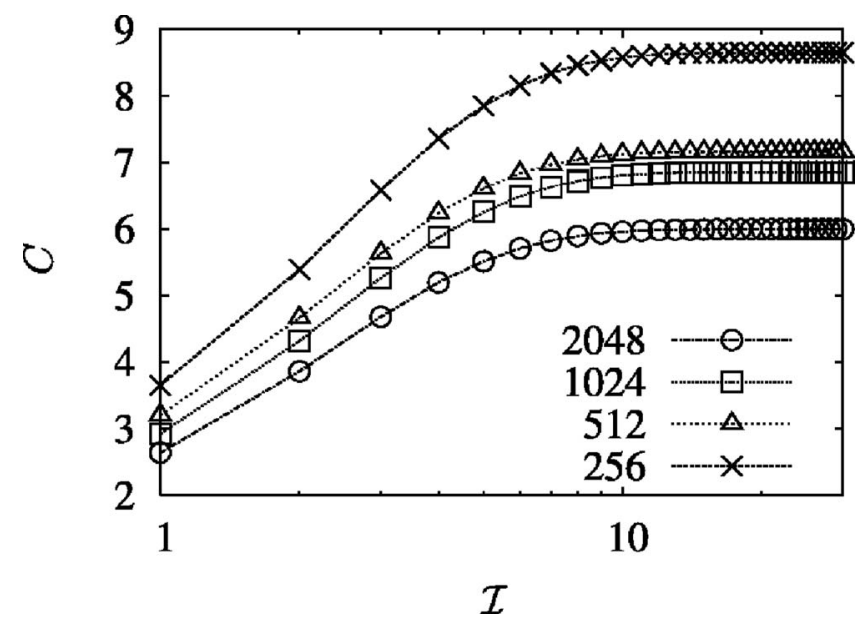

FIG. 15. Compression rate $C$ vs number of iterations $\mathcal{I}$ for runs $256^{3}, 512^{3}$, $1024^{3}$, and $2048^{3}$.

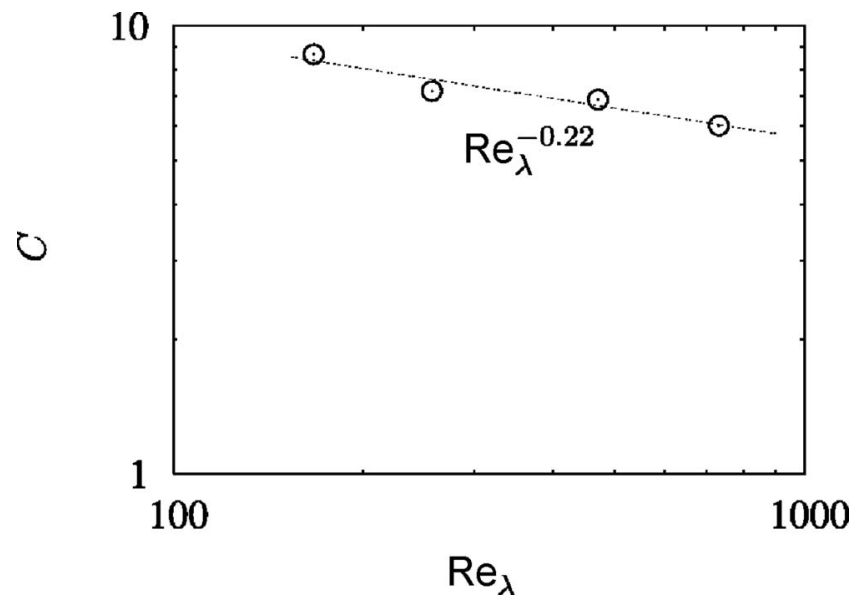

FIG. 16. Compression rate after convergence of the CVE algorithm vs $\operatorname{Re}_{\lambda}$. The exponent is estimated by a least-square fit of the four available data points.
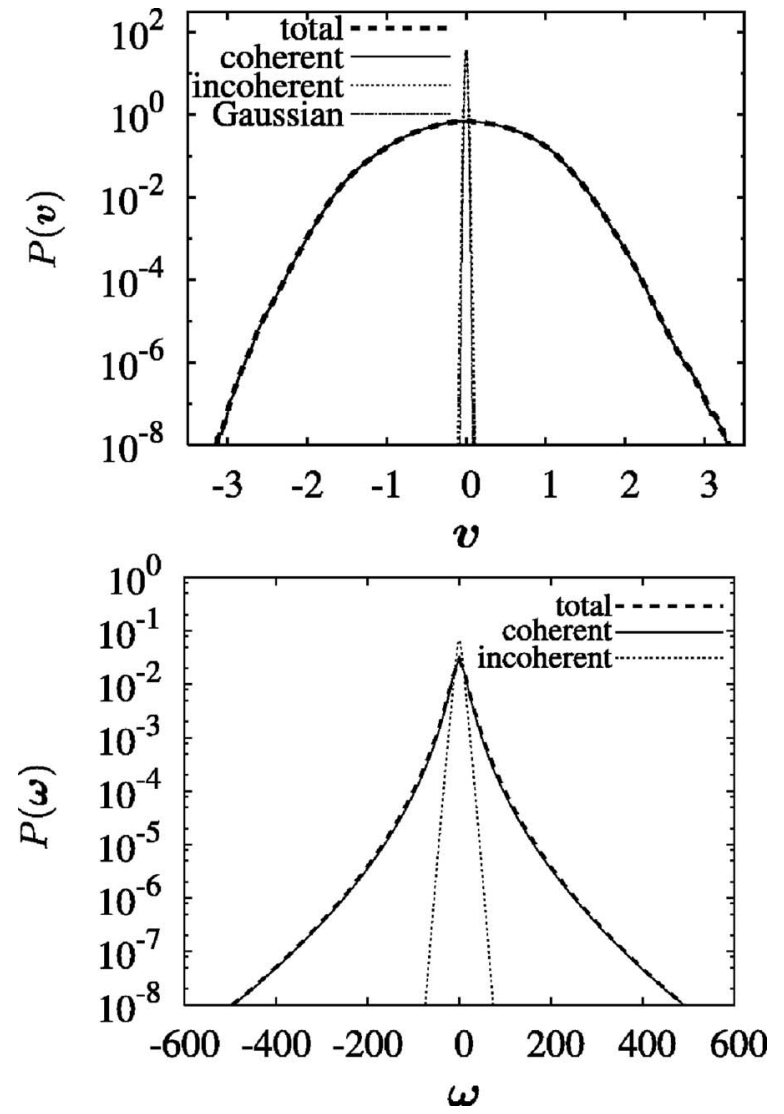

FIG. 17. PDFs of velocity (top) and vorticity (bottom) after the convergence for $\operatorname{Re}_{\lambda}=732$ and $N=2048^{3}$. The Gaussian distribution in the top figure is normalized so that it has zero mean and the same standard deviation as that of the incoherent velocity.

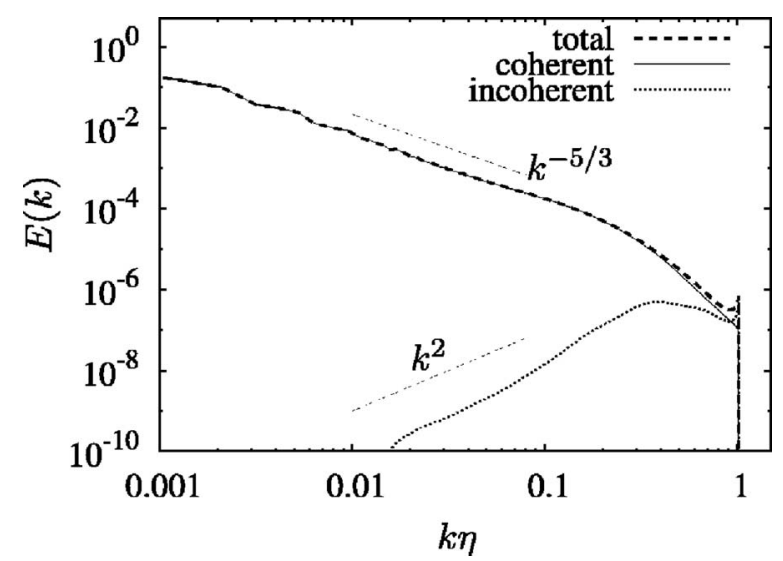

FIG. 18. Energy spectra of the total, coherent and incoherent flow after the convergence for $\operatorname{Re}_{\lambda}=732$ and $N=2048^{3}$. 
${ }^{1}$ S. A. Orszag, "Numerical methods for the simulation of turbulence," Phys. Fluids 12, 250 (1969).

${ }^{2}$ G. S. Patterson and S. A. Orszag, "Spectral calculations of isotropic turbulence: Efficient removal of aliasing interactions," Phys. Fluids 14, 2538 (1971).

${ }^{3}$ M. Yokokawa, K. Itakura, A. Uno, T. Ishihara, and Y. Kaneda, "16.4Tflops direct numerical simulation of turbulence by a Fourier spectral method on the Earth Simulator," in ACM/IEEE SC 2002 Conference (SC'02) (2002), p. 50.Baltimore, 2002 (http://www.sc-2002.org/paperpdfs/ pap.pap273.pdf).

${ }^{4}$ Y. Kaneda, T. Ishihara, M. Yokokawa, K. Itakura, and A. Uno, "Energy dissipation rate and energy spectrum in high resolution direct numerical simulations of turbulence in a periodic box," Phys. Fluids 15, L21 (2003).

${ }^{5}$ G. I. Taylor, "The spectrum of turbulence," Proc. R. Soc. London, Ser. A 164, 476 (1938).

${ }^{6}$ E. D. Siggia, "Numerical study of small-scale intermittency in 3-dimensional turbulence," J. Fluid Mech. 107, 375 (1981).

${ }^{7}$ A. Vincent and M. Meneguzzi, "The spatial structure and statistical properties of homogeneous turbulence," J. Fluid Mech. 225, 1 (1991).

${ }^{8}$ J. Jiménez, A. A. Wray, P. G. Saffman, and R. S. Rogallo, "The structure of intense vorticity in isotropic turbulence," J. Fluid Mech. 255, 65 (1993).

${ }^{9}$ A. Vincent and M. Meneguzzi, "The dynamics of vorticity tubes in homogeneous turbulence," J. Fluid Mech. 258, 245 (1994).

${ }^{10}$ S. Douady, Y. Couder, and M. E. Brachet, "Direct observation of the intermittency of intense vorticity filaments in turbulence," Phys. Rev. Lett. 67, 983 (1991).

${ }^{11}$ M. Farge and R. Sadourny, "Wave-vortex dynamics in rotating shallow water," J. Fluid Mech. 206, 433 (1989).

${ }^{12}$ M. Farge, "Wavelet transforms and their applications to turbulence," Annu. Rev. Fluid Mech. 24, 395 (1992).

${ }^{13}$ Wavelets in Physics, edited by J. C. van den Berg (Cambridge University Press, Cambridge, 1999).

${ }^{14}$ M. Farge and K. Schneider, "Analysing and computing turbulent flows using wavelets," in New Trends in Turbulence, Les Houches 2000, Vol. 74, edited by M. Lesieur, A. Yaglom, and F. David (Springer, New York, 2002, p. 449).

${ }^{15}$ M. Farge and K. Schneider, "Wavelets: applications to turbulence," in Encyclopedia of Mathematical Physics, edited by J. P. Françoise, G. Naber, and T. S. Tsun (Elsevier, Amsterdam, 2006), p. 408.

${ }^{16}$ M. Farge, K. Schneider, and N. Kevlahan, "Non-Gaussianity and coherent vortex simulation for two-dimensional turbulence using an adaptive orthonormal wavelet basis," Phys. Fluids 11, 2187 (1999).

${ }^{17}$ D. Donoho and I. Johnstone, "Ideal spatial adaptation via wavelet shrinkage," Biometrika 81, 425 (1994).

${ }^{18}$ M. Farge, G. Pellegrino, and K. Schneider, "Coherent vortex extraction in 3d turbulent flows using orthogonal wavelets," Phys. Rev. Lett. 87, 054501 (2001).

${ }^{19}$ D. E. Goldstein and O. V. Vasilyev, "Stochastic coherent adaptive large eddy simulation method," Phys. Fluids 16, 2497 (2004).

${ }^{20} \mathrm{O}$. Roussel, K. Schneider, and M. Farge, "Coherent vortex extraction in 3D homogeneous turbulence: comparison between orthogonal and biorthogonal wavelet decompositions," J. Turbul. 6, 11 (2005).

${ }^{21}$ M. Farge and K. Schneider, "Coherent vortex simulation (CVS), a semideterministic turbulence model using wavelets," Flow, Turbul. Combust. 66, 393 (2001).

${ }^{22}$ K. Schneider, M. Farge, G. Pellegrino, and M. Rogers, "Coherent vortex simulation of 3D turbulent mixing layers using orthogonal wavelets," J. Fluid Mech. 534, 39 (2005).

${ }^{23}$ K. Schneider, M. Farge, A. Azzalini, and J. Ziuber, "Coherent vortex extraction and simulation of 2D isotropic turbulence," J. Turbul. 7, 44 (2006).

${ }^{24}$ K. R. Sreenivasan, "Possible effects of small-scale intermittency in turbulent reactive flows," Flow, Turbul. Combust. 72, 115 (2004).

${ }^{25}$ S. Mallat, A Wavelet Tour of Signal Processing (Academic, New York, 1998).

${ }^{26}$ K. Schneider, M. Farge, and N. Kevlahan, "Spatial intermittency in twodimensional turbulence: a wavelet approach," in Woods Hole Mathematics, Perspectives in Mathematics and Physics, edited by N. Tongring and R. C. Penner (World Scientific, Singapore, 2004), Vol. 34, p. 302.

${ }^{27}$ A. Azzalini, M. Farge, and K. Schneider, "Nonlinear wavelet thresholding: A recursive method to determine the optimal denoising threshold," Appl. Comput. Harmon. Anal. 18, 177 (2005).

${ }^{28} \mathrm{Y}$. Kaneda and T. Ishihara, "High-resolution direct numerical simulation of turbulence," J. Turbul. 7, 20 (2006).

${ }^{29}$ K. R. Sreenivasan and R. A. Antonia, "The phenomenology of small-scale turbulence," Annu. Rev. Fluid Mech. 29, 435 (1997).

${ }^{30}$ M. Farge, K. Schneider, G. Pellegrino, A. Wray, and B. Rogallo, "Coherent vortex extraction in three-dimensional homogeneous turbulence: Comparison between CVS-wavelet and POD-Fourier decompositions," Phys. Fluids 15, 2886 (2003). 